\title{
Tangence
}

\section{Langue et urbanité dans la littérature québécoise}

\section{Marie-Andrée Beaudet}

Numéro 48, octobre 1995

Montréal et Vancouver : parcours urbains dans la littérature et le cinéma

URI : https://id.erudit.org/iderudit/025862ar

DOI : https://doi.org/10.7202/025862ar

Aller au sommaire du numéro

Éditeur(s)

Tangence

ISSN

0226-9554 (imprimé)

1710-0305 (numérique)

Découvrir la revue

Citer cet article

Beaudet, M.-A. (1995). Langue et urbanité dans la littérature québécoise.

Tangence, (48), 56-64. https://doi.org/10.7202/025862ar d'utilisation que vous pouvez consulter en ligne.

https://apropos.erudit.org/fr/usagers/politique-dutilisation/ 


\section{Langue et urbanité dans la littérature québécoise}

\section{Marie-Andrée Beaudet}

Avant même les singularités d'un thème, le dessin particulier d'un sentiment ou d'un décor, la langue littéraire se trouve chargée d'une expérience individuelle et collective d' être au monde". À travers la langue - le choix des mots, leur agencement, les référents qui les portent et indiquent non un sens fermé mais une voie vers le sens - tout un horizon socio-discursif s'exhibe. Au Québec, la question des rapports entre la langue et la littérature revêt, on le sait, une grande importance. Elle se double, pour ainsi dire, d'un facteur multiplicateur. La situation particulière du français au Québec pose d'une façon exacerbée les lois générales déjà définies par Bakhtine ${ }^{1}$ qui veulent que la langue fasse corps avec le social. Analysant la production littéraire québécoise et les relations de l'écrivain à sa langue maternelle, Lise Gauvin a parlé, avec raison, d'un phénomène de surconscience linguistique ${ }^{2}$ qui caractériserait non seulement une génération d'écrivains mais l'ensemble des générations d'écrivains qui se sont succédé au Québec. Déjà dans l'œuuvre de Michel Bibaud ${ }^{3}$, premier poète québécois à publier en terre canadienne, on peut voir des traces de cette surconscience linguistique :

La paresse nous fait mal parler notre langue

Combien peu, débitant la plus courte harangue

Savent garder et l'ordre, et le vrai sens des mots

1 Mikhail Bakhtine (V. N. Volochinov), Le marxisme et la philosopbie du langage. Essai dapplication de la méthode sociologique en linguistique, Paris, Minuit, coll, "Le sens commun", 1977, 233 p. [1929]. Bakhtine y écrivait que " la langue ne saurat être définie comme un produit fini, qu'en fait elle ne se transmet pas, [çu']elle dure sous la forme d'un processus d'évolution ininterrompu [...] et [que] la langue maternelle n'est pas acquise par les individus [que] c'est en elle et par elle qu'a lieu leur premier éveiln.

2 Lise Gauvin, "La surconscience linguistique de l'écrivain francophone: positions des revues francophones", Colloque de Bruxelles, mai 1991.

3 Michel Bilsaud, Fittres, satires, chansons, épigrammes et autres piéces en vers, Montréal, Ludger Duvernay, $1830,178 \mathrm{p}$. 
Au critique français Isidore Lebrun ${ }^{4}$ qui lui reproche des incohérences de style, Bibaud répondra, engageant un autre débat qui aura une longue fortune, que, s'il est vrai que son art manque de précision, son ouvre n'est destinée qu'à ses compatriotes et non à des lecteurs européens. Cette première querelle linguistique amorce toute une série de débats sur la qualité du français parlé et écrit au Canada, qui marquent autant l'histoire littéraire du XIx ${ }^{\mathrm{e}}$ siècle que celle du siècle suivant. À divers moments, des changements de conjoncture sociale ont provoqué la redéfinition des termes du débat linguistico-littéraire. Au nombre de ces changements de conjoncture, il faut compter l'expérience concrète de la ville.

Dans le cadre de cette problématique mettant en rapport l'urbanité, la langue et la littérature, deux périodes de l'histoire littéraire du Québec présentent un intérêt particulier. La première ouvre le $\mathrm{xx}^{\mathrm{e}}$ siècle et la seconde se situe dans les premières années de la Révolution tranquille. Dans les deux cas, mais d'une façon inversée, la ville (Montréal) se retrouve au cœur des enjeux de la définition de la langue littéraire.

\section{Montréal et les villes-jardins}

Sans vouloir reprendre toute l'histoire de la longue querelle qui opposa les régionalistes et les exotiques, déjà bien analysée par Annette Hayward ${ }^{5}$, j'insisterai sur un aspect souvent négligé, celui de ses fondements linguistiques.

J'ai mis en évidence, dans une recherche antérieure ${ }^{6}$, l'antagonisme qui, en matière de langue, marque les positions des deux esthétiques. Le tableau suivant permet de schématiser les caractères valorisés de part et d'autre:

4 Isidore Lebrun, article paru dans La Revue encyclopédique, Paris, 1830.

5 Annette Hayward, "Le conflit entre les régionalistes et les "exotiques" au Québec (1900-1920)", thèse de doctorat, Montréal, Université McGill, 1980, $1046 \mathrm{p}$.

6. Marie-Andrée Beaudet, Langue et littérature au Québec, 1895-1914. L'impact de la situation linguistique sur la formation du champ littéraire, Montréal, l'Hexagone, coll. "Essais littéraires ", 1991, 221 p. Le tableau reproduit dans le présent article provient de cette étude. 


\begin{tabular}{|l|l|}
\hline \multicolumn{2}{|l|}{ PRINCIPAUX CARACTÈRES DE LA LANGUE VALORISÉS PAR } \\
\hline LES RÉGIONALISTES & LES EXOTIQUES \\
\hline rurale, paysanne & urbaine \\
\hline populaire & savante \\
\hline concrète & abstraite \\
\hline provincialiste & parisianiste \\
\hline orale & écrite \\
\hline simple, naturelle & rare, travaillée \\
\hline impersonnelle, collective & subjective \\
\hline archaïque & nouvelle \\
\hline classique (xvi ${ }^{\mathrm{e}}$ siècle) & moderne \\
\hline "canadianiste" & "néologiste" \\
\hline
\end{tabular}

On ne s'étonne guère de voir le premier groupe d'exotiques, composé des poètes Paul Morin, Guy Delahaye, René Chopin et du critique Marcel Dugas (qui publieront tous leurs premiers livres entre 1910 et 1913), défendre une langue plus moderne et plus urbaine lorsque l'on met en parallèle leurs positions et leurs trajectoires familiales et scolaires. Comme le souligne l'historien Robert Lahaise: "nombre d'éléments concordait à les réunir. Tous les quatre sont de familles relativement aisées et sont passés par le moule privilégié du collège classique ${ }^{7}$. Morin, Chopin et Delahaye se rencontrent au Collège Sainte-Marie et Dugas se joint à eux au début de leurs études universitaires à l'Université Laval de Montréal. Tous les quatre sont nés à Montréal ou dans la région de Montréal. D'origine bourgeoise, ils avaient hérité de leur milieu familial un "habitus" ${ }^{8}$ les disposant à reconnaître au monde de l'Art une valeur prépondérante. L'ouvre de Nelligan, poète montréalais par excellence, constitue pour eux un phare. Ils y retrouvent une sensibilité, un idéal et une esthétique qui rejoignent les leurs.

7 Robert Lahaise, Guy Delahaye et la modernité littéraire, Montréal, Hurtubise $\mathrm{HMH}$, coll. "Cahiers du Québec/littérature", 1987, p. 62.

8 Au sens où l'emploie Pierre Bourdieu. 
Aux mots d'ordre de Camille Roy et de la Société du parler français au Canada qui plaidaient pour la constitution d'une littérature nationale définie comme essentiellement rurale et catholique et valorisant l'emploi d'une langue populaire, les exotiques opposent la théorie de l'Art pour l'Art qui, autant en France qu'au Québec, du moins en poésie, renvoie à une expérience de la ville et de la modernité. Walter Benjamin a montré comment la ville de Paris et son paysage urbain informent la démarche poétique de Baudelaire. Au Québec, l'œuvre de Guy Delahaye, en particulier "Mignonne allons voir si la rose...", qui présente les caractères d'un véritable manifeste poétique, se construit en opposition déclarée à l'esthétique régionaliste ${ }^{9}$. Rejetant la thématique ruraliste et le niveau de langue "naturelle" qu'illustrait à merveille, aux yeux des critiques de l'époque, le recueil d'Englebert Gallèze [Lionel Léveillée] paru la même année que les Phases (1910) et intitulé Les chemins de l'âme, Delahaye raille et met à mal les naïvetés et les maladresses formelles de son rival en émaillant ses poèmes-répliques de termes spécialisés empruntés au vocabulaire moderne de la pharmacologie et de la médecine. Le jeune poète démontre qu'

On peut ne pas faire dans le genre patriotico-religieux-abruti-traditionnel ${ }^{10}$

Étrangement, les poètes qui feront bloc contre une vision de la littérature privilégiant un espace géographique et linguistique centré sur la ruralité s'intéresseront peu à Montréal. Nelligan, dont la vie - comme le rappelle Michel Biron dans un texte récent ${ }^{11}$ - s'inscrit si fortement dans le paysage montréalais, ne lui consacrera qu'un poème et l'un de ses moins achevés: "NotreDame-des Neiges" "2. Paul Morin, dans Poèmes de cendre et d'or, ne fera qu'une brève allusion à Montréal :

9 Voir mon article "Mignonne, allons voir si la rose..." de Guy Delahaye: intertextualité et champ littéraire", Études françaises, vol. XXIX, n 1, 1993, p. 127-134́.

10 Guy Delahaye, "Mignonne, allons voir si la rose...", dans Robert Lahaise (dir.), Guy Delahaye: CEuvres parues et inédites, Montréal, Hurtubise HMH, coll. "Cahiers du Québec/textes et documents", 1988, p. 212.

11 Michel Biron, "La romance du libéralisme: poésie et roman au tournant du siècle" Montréal imaginaire. Ville et littérature, sous la direction de Pierre Nepveu et Gilles Marcotte, Montréal, Fides, 1992, p. 149-209.

12 Émile Nelligan, Poésies complètes, 1896-1899, Montréal, Fides, 1952, p. 148149. 
60

Poète, prends ton luther, ton portefeuille vert,

Ton stylograpbe Waterman, et le tramway

Qui rampe vers la Place d'Armes

Comme un mastodonte enroué... ${ }^{13}$

Chez les poètes modernistes du début du siècle, les villes dont on parle sont des villes que l'on visite, où l'on est de passage et qui invitent davantage au dépaysement qu'à l'inscription. Des villes qui sont réduites très souvent à des parcs, à des jardins. Chez Morin, comme chez Chopin, abondent les figures et les thèmes reliés à la Nature:

Nature, ce matin, vous m'avez fait du mal

Je n'affronterai plus le jardin estival

Où vos doigts parfumés de menthe et d'ancolie

Versent moins de plaisir que de mélancolie ${ }^{14}$

C'est encore à une métaphore associée à la Nature que Morin puisera sa comparaison entre la France et le Québec, entre Paris et Montréal, dans le poème qui clôt Le paon d'émail et qui s'intitule laconiquement "À ceux de mon pays":

J'attends d'être muri par la bonne souffrance

Pour, un jour, marier

Les mots canadiens aux rythmes de France

Et l'érable au laurier ${ }^{15}$

C'est à l'intérieur même de la thématique privilégiée par les régionalistes que les exotiques inscriront leur dissidence. La Nature n'est plus pour eux celle qui nourrit, celle du laboureur et du paysan, mais celle, au contraire, qui dépayse le voyageur et qui le repayse dans le monde qu'il a élu, celui de la Culture et des livres. C'est à une Nature modélisée par l'Art et la mythologie que s'intéressent les exotiques. En définitive, l'esthétique qui est la leur n'impose pas tant le choix d'une autre thématique que le choix d'une distance, celle de l'étrangeté et de l'abstraction. Distance rendue sensible par le travail de la forme et l'adoption de mots rares évoquant des horizons venus d'ailleurs mais inséparables de l'expérience concrète de la ville.

13 Paul Morin, "La revanche du Paon" (dans Poème de cendre et d'or), CEures poétiques, Montréal, Fides, 1961, p. 195.

14 Paul Morin, "Nature, ce matin..." Le paon d'émail, ibid., p. 71.

15 Ibid., p. 147. 


\section{Montréal : la ville des autres}

Autant le Montréal de Nelligan et des premiers exotiques est une ville bourgeoise qui favorise l'ouverture sur le monde, autant le Montréal des écrivains réunis autour de la revue Parti pris est une ville prolétaire, marquée de tous les signes de la fermeture et de l'aliénation. Dans un cas, Montréal parle la langue des privilégiés de la culture, dans l'autre, elle parle la langue de la "dépossession du monde", pour reprendre le titre d'un ouvrage de Jacques Berque.

Pour les partipristes comme pour les exotiques, Montréal représente le lieu premier de formation et de rencontre. Comme le rappelait Robert Major au Colloque du Mont-Gabriel sur "Montréal et son destin littéraire":

Les animateurs de Parti pris [...] sont tous Montréalais. Le noyau fondateur, André Major, André Brochu, Pierre Maheu, Jean-Marc Piotte, Paul Chamberland, a tissé des liens au Collège Sainte-Marie ou à l'Université de Montréal [...]. L'Université de Montréal, qu'on y soit étudiant en philosophie, en lettres, en sociologie, en sciences politiques, ou jeune professeur, comme André Brochu, est le vestibule de la revue. ${ }^{16}$

On le voit, les circonstances de formation du groupe offrent beaucoup de similitudes avec celles des exotiques. Mais à une distinction près: les partipristes sont issus de milieux familiaux beaucoup plus modestes. Cette détermination, ajoutée à leurs lectures politiques, fera toute la différence. La ville qu'ils connaissent et qui les inspire est la ville des petits travailleurs de l'Est.

Dans un numéro intitulé "Montréal, ville des autres", Pierre Maheu écrit :

En fait il y a deux villes, deux mondes qui coexistent à Montréal, l'un à l'est, français, pauvre, c'est la ville indigène; l'autre à l'ouest, riche, anglais, dont une partie s'appelle The Town, c'est ce qu'on appelle le biculturalisme. Le contact de ces deux mondes n'a pas été fructueux; l'est est sale, on y souffre de promiscuité, les maisons y sont en pleine décrépitude, comme la langue qu'y parlent les gens, langue pauvre comme leur vie, comme leurs sentiments qui ne peuvent être que résignation ou colère; l'ouest est cossu, aéré, les maisons fermées

16 Robert Major, "Parti pris montréalais", Colloque "Montréal et son destin littéraire ", Montréal, Écrits du Canada français, no 76, 1992, p. 131. 
62

sur elles-mêmes, on y est individualiste, tout y respire la morgue et la suffisance, avec, à l'arrière-plan, un certain malaise: car parfois, les boites aux lettres explosent. ${ }^{17}$

Les cuvres issues du groupe reprendront ce portrait divisé de Montréal, divisé dans son espace géographique et dans sa langue. Dans La ville inbumaine de Laurent Girouard ${ }^{18}$ ou dans Le cassé de Jacques Renaud ${ }^{19}$, Montréal apparaît comme un lieu de violence et d'impuissance.

"Montréal grand comme un désordre universel ", écrit Gaston Miron. "Montréal à cadence de désastre à la trogne d'un ivrogne", clame Paul Chamberland dans les premiers extraits de L'afficheur burle qui sont publiés dans le même numéro de la revue Parti pris auquel nous venons de faire allusion ${ }^{20}$. Pour les animateurs du groupe, Montréal est plus que Montréal. La ville est, comme le dit encore Robert Major dans le texte déjà cité, "la vitrine où se voient, de manière crue, les effets pervers d'une situation qui accable l'ensemble de la collectivité "21. Le joual, phénomène originellement montréalais, devient de la même façon l'expression de la misère de tout un peuple, "le décalque parfait de la décadence de notre culture", écrivait Gérald Godin dans Parti pris ${ }^{22}$.

L'expérience montréalaise se présente comme une expérience primordiale dans la formation de la pensée partipriste comme elle l'avait été dans celle des premiers poètes exotiques. Il est intéressant de mettre ici en parallèle les interrrogations qui ponctuent les analyses que Robert Major et Michel Biron ont consacrées respectivement au groupe des fondateurs du mouvement Parti pris et à la poésie du tournant du siècle. Robert Major écrit: "Peut-on concevoir un tel mouvement littéraire et politique [Parti pris] ailleurs qu'à Montréal, au début des années soixante?" 23 Et Michel Biron, à son tour, reprend l'interrogation: “Émile Nelligan

17 Pierre Maheu, "En guise d'introduction", Parti pris ("Montréal, ville des autres"), vol. II, $\mathrm{n}^{\circ} 4$, décembre 1964 , p. 10.

18 Laurent Girouard, La ville inbumaine, Montréal, Parti pris, 1964, 187 p.

19 Jacques Renaud, Le cassé, Montréal, Parti pris, 1964, 126 p.

20 Paul Chamberland, "L'afficheur hurle", Parti pris, vol. II, $\mathrm{n}^{\circ} 4$, décembre 1964 , p. 52.

21 Robert Major, "Parti pris montréalais ", op. cit., p. 136.

22 Gérald Godin, "Le joual et nous", Parti pris, vol. II, no 5, janvier 1965, p. 18.

23 Robert Major, "Parti pris montréalais", p. 130. 
pouvait-il écrire "La romance du vin" ailleurs qu'à Montréal et à un autre moment qu'au tournant du $\mathrm{Xx}^{\mathrm{e}}$ siècle? ${ }^{24}$

À cinquante ans d'intervalle, Montréal offre à deux groupes de jeunes écrivains l'expérience de la confrontation des cultures et du choc des langues. Chez les exotiques, la rupture prendra la forme d'une contestation de la vision traditionnellement rurale du Québec à un moment où l'industrialisation et l'urbanisation ont déjà transformé la réalité sociale et linguistique et provoqué l'apparition des formes modernes d'une nouvelle bourgeoisie canadienne-française. La célébration d'une langue hyper-soignée et savante comme la célébration des signes de la culture renvoient au déchirement d'une bourgeoisie qui ne trouve plus à s'exprimer dans les "maigres mots frileux de l'héritage" 25 et qui ne parvient pas encore à nommer les réalités de la ville familière.

Chez les partipristes, l'élection stratégique - il convient de le rappeler - d'une "langue humiliée" comme langue littéraire correspondait à une lecture non plus esthétique du monde, mais à une lecture politique. Lecture qui met en scène Montréal comme lieu emblématique de l'aliénation de tout un peuple. Les œuvres des deux groupes, que pourtant tout sépare, témoignent d'un phénomène diglossique. J'entends ici "diglossie" au sens où le linguiste André Martinet la définit, c'est-à-dire comme une situation où un groupe ou une communauté "utilise, selon les circonstances, un idiome plus familier et de moindre prestige ou un autre plus savant et plus recherché ${ }_{\text {. }}{ }^{26}$.

La diglossie à l'œuvre chez les exotiques oppose deux variétés de français, le français littéraire importé d'Europe et le français canadien rural valorisé par les régionalistes, alors que la diglossie portée par le joual des partipristes met l'accent sur la domination exercée par la langue des dominants, l'anglais, sur la langue maternelle des Québécois.

Charles ab der Halden écrivait en 1907: "Il est peu de questions aussi capitales pour le Canada français que celle de la

24 Michel Biron, "La romance du libéralisme: poésie et roman au tournant du siècle , p. 149.

25 Gaston Miron, L'bomme rapaillé, Paris, Maspero, 1981, p. 94.

26 André Martinet, Éléments de linguistique générale, Paris, Armand Colin, coll. "U, 1967, p. 148. 
64

langue " 27 . Toute l'histoire littéraire du $\mathrm{xx}^{\mathrm{e}}$ siècle allait lui donner raison en trouvant dans Montréal un lieu propice à l'éclosion des tensions qui habitaient et habitent peut-être toujours la réalité et l'imaginaire québécois.

27 Charles ab der Halden, Nouvelles études de littérature canadienne française, Paris, F. R. de Rudeval, 1907. 